\title{
Impact absorption measurement of a gel sheet using an optical interferometer
}

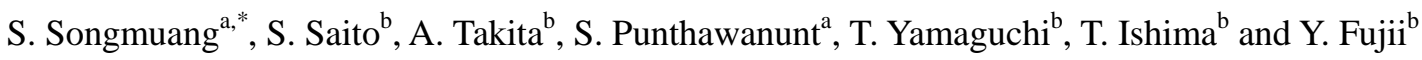 \\ ${ }^{a}$ Faculty of Science and Technology, Kasem Bundit University, 1761 Pattanakarn Rd. Suanluang, Bangkok 10250, \\ Thailand \\ ${ }^{\mathrm{b}}$ Faculty of Science and Technology, Gunma University, Kiryu, Tenjin-cho 1-5-1, 376-8515, Japan \\ *sa-nga.son@kbu.ac.th
}

\begin{abstract}
In this paper, the Doppler shift of light is used to manipulate the change in optical path length using the classical interferometer. From which, the change in optical path length is related to the change in impact force, where finally the last of impact force can be related to the gel sheet absorption.
\end{abstract}

Keywords: Impact absorption, Optical interferometer, Measurement.

\section{Introduction}

Over the past few years the increasing focus of the structures impact loading was proposed by several researches [1-10], such as, the force is measured highly accurately as inertial force acting on a mass [11-16], using an optical interferometer $[17,18]$. An optical interferometer was used to accurately measure the velocity $[19,20]$. There have been works more accuracy technology than classical interferometer [21-23]. Because of optical fiber sensors have been widely used in structural health monitoring and damage identification [24] due to its ability of sensing the importance structural mechanical parameters such as position, strain, stress, curvature, temperature, and etc. Furthermore, there also have been proposed the impact force and impact energy absorption tests for mouthguards have used a steel ball in a drop ball $[25,26]$ and a method for measuring the impact force of a spherical body dropping onto a water surface, which this paper refer to the method by modifying [27].

Impact is a complex event involving several phenomena. Furthermore, the nature of impact response influences the type of damage and the extent of structural degradation. Extensive researches have been carried out concerning the impact behavior of composite materials [28]. Dynamic behavior of materials is focusing on the analysis and design of energy absorbing materials and structures. The work presented that the inertia of the composite plays a very important role in absorbing energy, which can be reduced significantly due to impact damage caused. Thus, there have been several of experiments investigations concerning the energy-absorption materials [29-32], which the interesting point at the effective way is to reduce impact pressure and other unexpected damage [33-35].

Gel sheets have been widely used in various materials due to their unique and impact resistant properties such as high elasticity and viscoelasticity [36-38]. Therefore, get sheets are very important for industrial automotive that are capable of safely withstanding significant impacts, thermal gel sheets that are dissipation of heat in electronic components, medical scar gel sheets, mat industry, and various capable for reducing extreme shock, vibration environments, high damping, protection for fragile components from earthquake effects, seat cushion and etc. Thus, the accurate measurement of a tensile strain or compressive stress plays a crucial role in many research and industrial field to create a wide range of innovative products. This has led to considerable research being carried out on impact energy absorbers. The focus of this research has been on the force acting of a metal ball onto get sheet is measured using an optical interferometer. The acceleration, displacement, and interval of the sphere are calculated from the velocity of the center of gravity of a metal ball. The light source used was a He-Ne laser in which the two wavelengths $[39,40]$. A high-speed camera is 
used to capture the images around the impact region, which shows the changes with the impact force. However, it is difficult to determine the uncertainty in measuring varying force acting onto the material, which is the impact absorption measurement. Thus, we are investigated in a synchronization and obtained the advantage of the product and acceleration with the force is directly calculated according to its.

In this paper, we investigated the impact absorption measurement of a gel sheet using an optical interferometer, and its validity is experimentally proposed. Results have shown in the Figures 2-5.

\section{Experiment}

We investigated a method for measuring the impact absorption of a metal ball by a vertical dropping $(15.5 \mathrm{~cm}$. drop height) onto a gel sheet, which is in $5 \mathrm{~mm}$ thickness and in 10x10 cm sheet size. Blue and transparent gel sheets with a sticky surface, it is elastic and very resistant to compression, covered on both sides with thin and supple polyurethane films were selected for the experiment. The total mass of the metal ball, $M$, is $0.09388 \mathrm{~kg}$, and $30.2 \mathrm{~mm}$ in diameter was constant of all 20 sets performed, which are 10 sets for the blue get sheet and 10 sets for the other by the acceleration due to gravity. A cube corner prism, $12.7 \mathrm{~mm}$ in diameter, is inserted with an adhesive agent so that its optical center coincides with the center of gravity of the whole body. An optical interferometer is used to accurately measure the velocity. The force of impact and velocity against time was recorded using a high-speed camera for the interference stripe in real time with a resolution of 38400 pixels and a frame rate of $1200 \mathrm{fps}$.

Figure 1 shows a detailed schematic diagram of the experimental setup for testing the impact absorption measurement of a gel sheet using an optical interferometer. The digitizer and the high-speed camera are initiated by a trigger signal generated using DAC. This signal is activated by means of a light switch, which is a combination of a LD and PD that was switching manually. Impact occurred by releasing the metal ball from drop height $15.5 \mathrm{~cm}$, which it was manually. The velocity of the center of gravity of a metal ball, in which a cube corner prism is embedded so that its optical center co-insides with the center of gravity of the ball, is accurately measured using an optical interferometer with a sampling interval of approximately 1 ms. Computer is used to record and process the data by using the LabView program. The computer recorded the

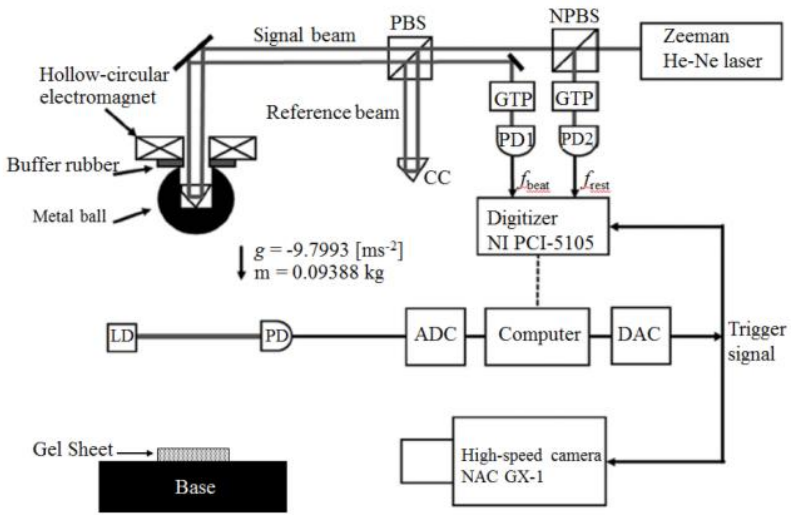

Fig. 1. A detailed schematic diagram of the experimental setup.

data in a rate of $30 \mathrm{M}$ (samples/s) and was set recorded length $5 \mathrm{M}$ samples number. When initiated, the program would begin continuously scanning data into a buffer until the trigger event occurred, then save samples along with 0.073 trigger (Th) samples to a tab delimited text file and then pass the data to the next state of the program for processing. The processed data were then saved as a separate tab delimited text file and further manipulation was done using CProgram and Microsoft Excel.

The total force acting on the metal ball is equivalent to the product of its mass and acceleration; i.e., $F_{\text {mass }}=M a$. The acceleration is calculated from the metal ball's velocity, and the velocity is calculated from the measured value of the Doppler shift frequency of the signal beam of interferometer $f_{\text {Doppler, }}$, which can be expressed as

$$
\begin{gathered}
v=\lambda_{\text {air }}\left(f_{\text {Doppler }}\right) / 2, \\
f_{\text {Doppler }}=-\left(f_{\text {beat }}-f_{\text {rest }}\right)
\end{gathered}
$$

where $\lambda_{\text {air }}$ is the wavelength of the signal beam, $f_{\text {beat }}$ is the beat frequency, and $f_{\text {rest }}$ is the frequency difference between the signal beam and the reference beam. The rest frequency $f_{\text {rest }}$ equivalent to the beat frequency $f_{\text {beat }}$ when the metal ball is at rest and no Doppler shift is added to the signal beam.

The total force, $F_{\text {mass }}$, consists of the gravitational force acting upon the metal ball, $-M g$, and the impact force acting from the gel sheet, $F_{\text {gelsheet, }}$ if other forces, such as the air drag and the magnetic force, are negligible. Then, the total force is

$$
F_{\text {mass }}=-M g+F_{\text {gelsheet }},
$$

where $g$ is the acceleration of gravity, approximately $9.799 \mathrm{~m} / \mathrm{s}^{2}$ at the experimental environment.

Therefore, the impact force acting from the gel sheet can be calculated as

$$
F_{\text {gelsheet }}=F_{\text {mass }}+M g \text {. }
$$


If other forces, such as air drag, cannot be ignored, then $F_{\text {gelsheet }}$ is assumed to include those other forces.

The velocity is accurately measured using an optical interferometer. The light source used was a Zeeman-type two wavelengths He-Ne laser had orthogonal polarization [39]. The difference between the two frequencies; i.e., the rest frequency $f_{\text {rest }}$, is approximately $3.0 \mathrm{MHz}$.

A digitizer records the output signals of PD1 and PD2 with a sample number of $5 \mathrm{M}$ for each channel, a sampling rate of $30 \mathrm{M}$ samples per second, and a resolution of 8 bit. The measurement duration of the digitizer is approximately $0.073 \mathrm{~s}$. The frequencies $f_{\text {beat }}$ and $f_{\text {rest }}$ are accurately determined from the digitized waveforms of the output signals appearing at PD1 and PD2, respectively, using the recently developed Zero-Crossing Fitting method (ZFM) [40]. In our analysis, the sampling interval is defined by $N$ $=500$ periods of the signal waveform, which corresponds to $0.125 \mathrm{~ms}$ when $f_{\text {beat }}$ is approximately $8 \mathrm{MHz}$.

The force of impact and velocity against time was recorded using a high-speed camera for the interference stripe in real time with a resolution of 38400 pixels and a frame rate of $1200 \mathrm{fps}$

In the experiment, was constant of all 20 sets performed, which are 10 sets for the blue get sheet and 10 sets for the transparent gel sheet. In each of the test, the metal ball was fixed onto the hollow-circular electromagnet that was held and released by turned on/off manually in a vertical drop height is approximately $15.5 \mathrm{~cm}$. The digitizer and the high-speed camera are initiated by a trigger signal that is generated using a DAC. This signal is activated by means of a light switch, which a combination of the LD and PD.

\section{Results}

Figure 2 shows the data processing procedure, which are calculated the frequencies $f_{\text {beat }}$ and $f_{\text {rest }}$ that is properly measured by the beat frequency for the velocity, $v$; the position, $x$; the acceleration, $a$; the total force acting upon the metal ball, $F_{\text {mass }}$; and the impact force acting onto the metal ball from the transparent gel sheet, $F_{\text {gelsheet }}$.

\section{Frequency}

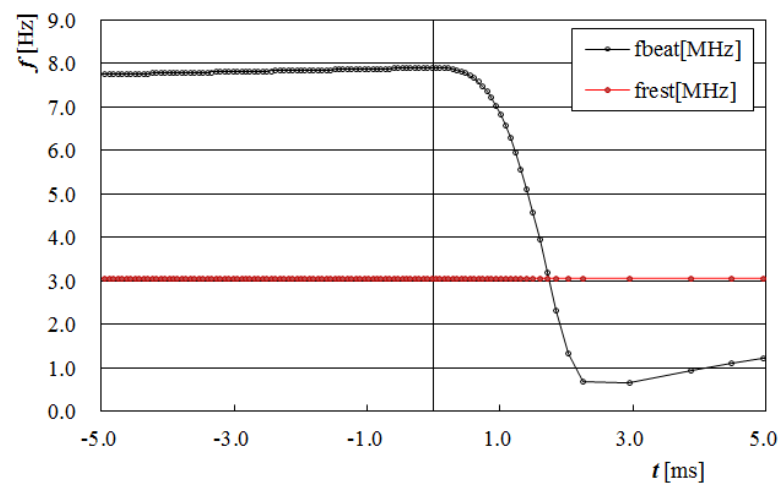

(a)

Velocity

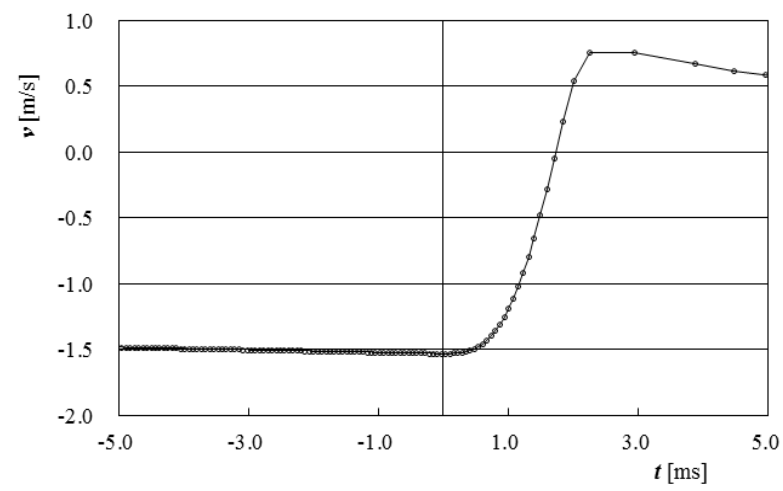

(b)

Displacement

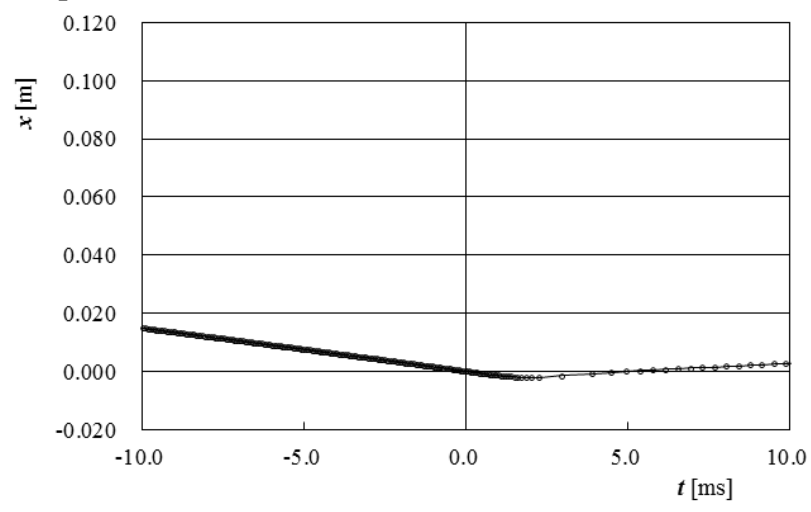

(c)

Acceleration

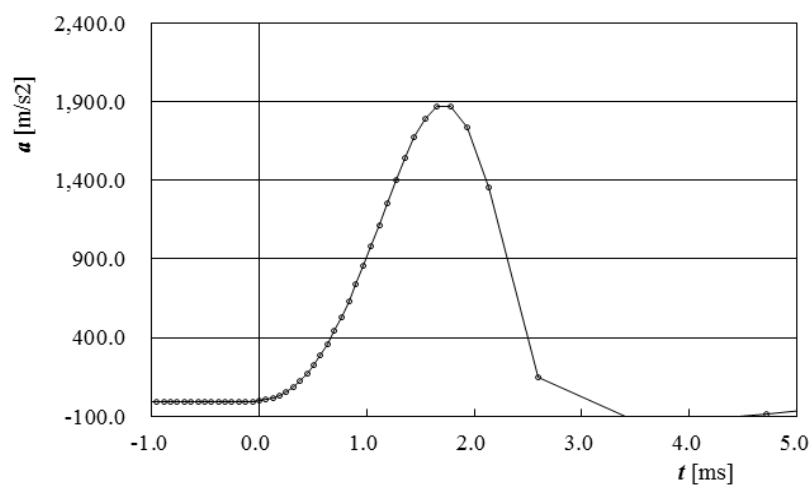

(d) 
Total force acting on the mass

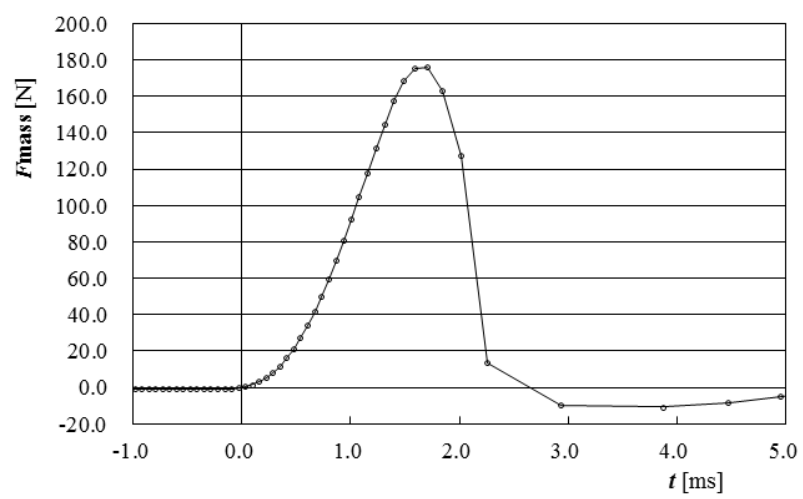

(e)

Total force acting from the gel sheet

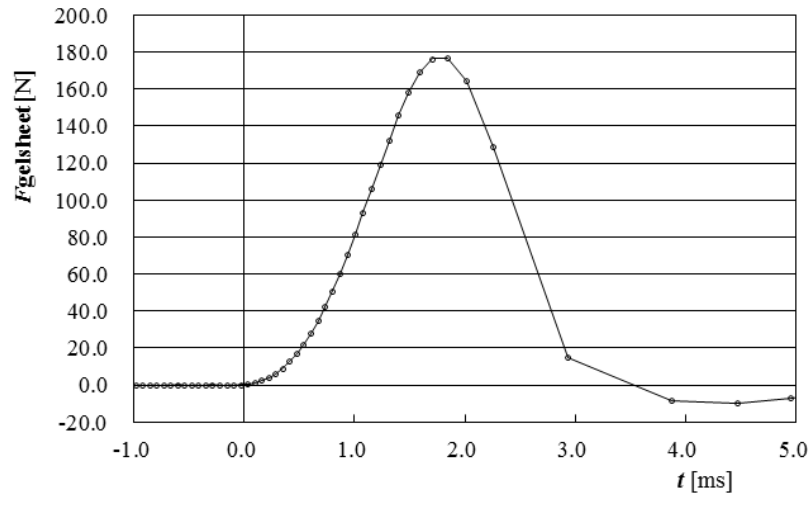

(f)

Fig. 2. (a-f) Data processing procedure: Calculation of velocity, position, acceleration, and force from frequency.

Figure 3 shows the drop test with the changes in impact force and velocity against time, which are captured images by the high-speed camera, in which, $F_{\text {gelsheet, and the }}$ velocity, $v$, against the time, $t$. When the metal ball impacts the gel sheet, $F_{\text {gelsheet }}$ at a drop of a hat to a maximum value of approximately $180.0 \mathrm{~N}$ at $t=1.7 \mathrm{~ms}$ and $x=-0.0021$ $\mathrm{mm}$.

Change in force against time

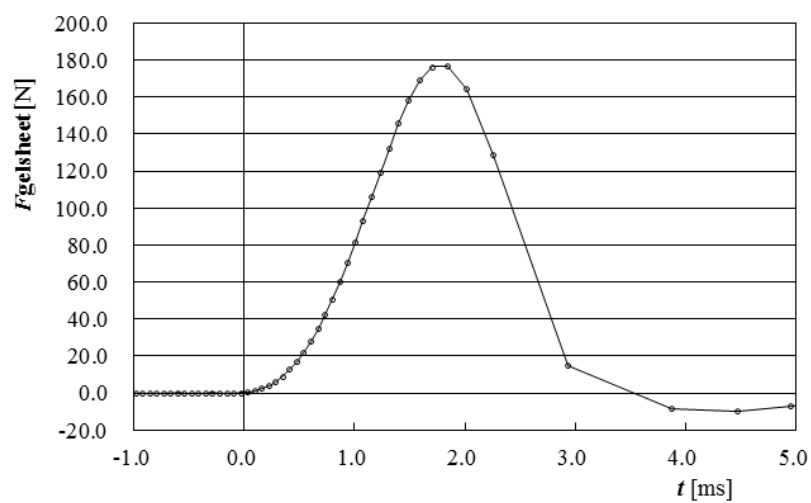

(a)
Total force acting on the mass

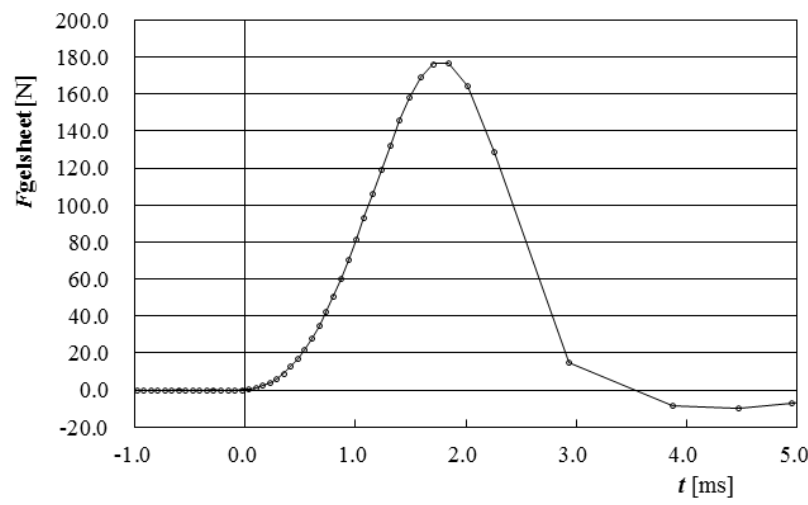

(b)

Total force acting from the gel sheet

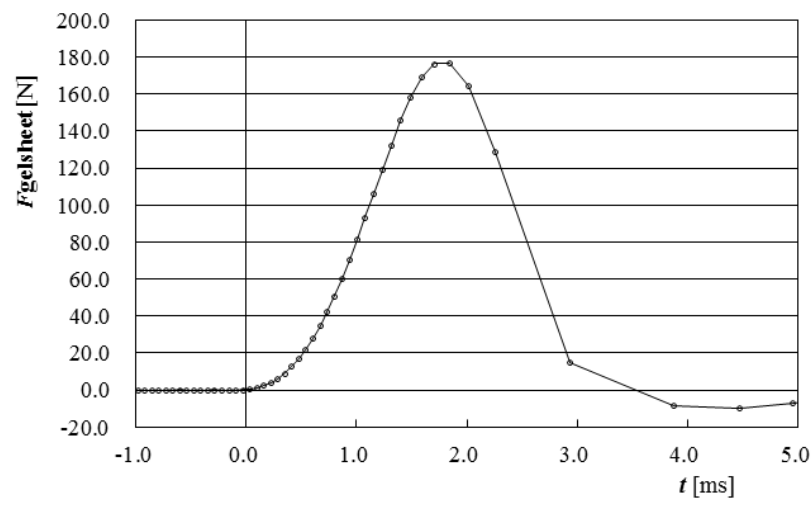

(c)

Fig. 3. (a-c) Changes in impact force and velocity against time.

Figure 4 shows the changes in $F_{\text {gelsheet }}$ against time for all the 10 drop measurements. The results of the 10 drop measurements coincide well, indicating a high reproducibility of the measurements.

Changes in force against time

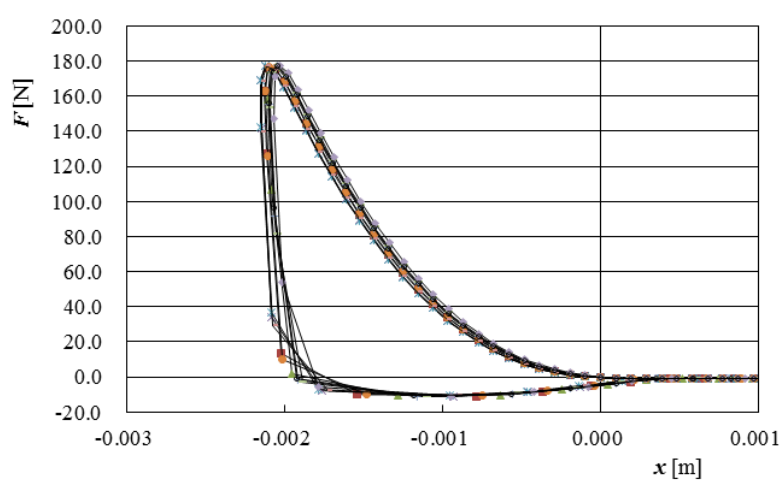

(a) 
Changes in force acting from the gel sheet

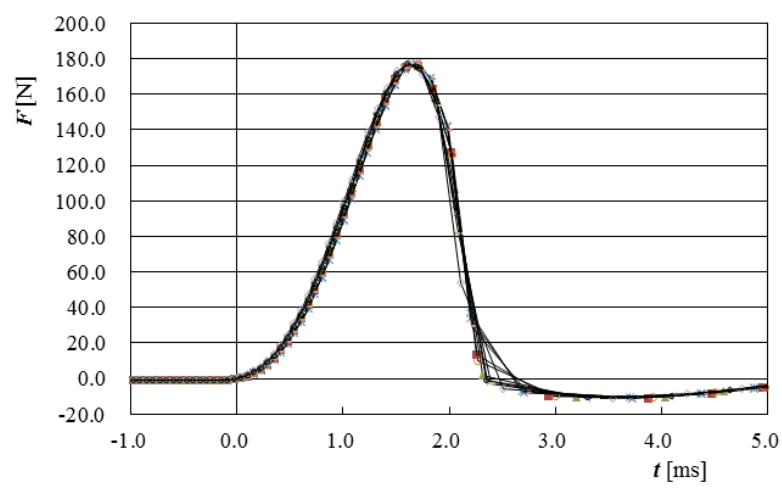

(b)

Fig. 4. (a, b) Changes in force against time and changes in force acting from the gel sheet (ten drop measurements).

Changes in impact force and velocity against time

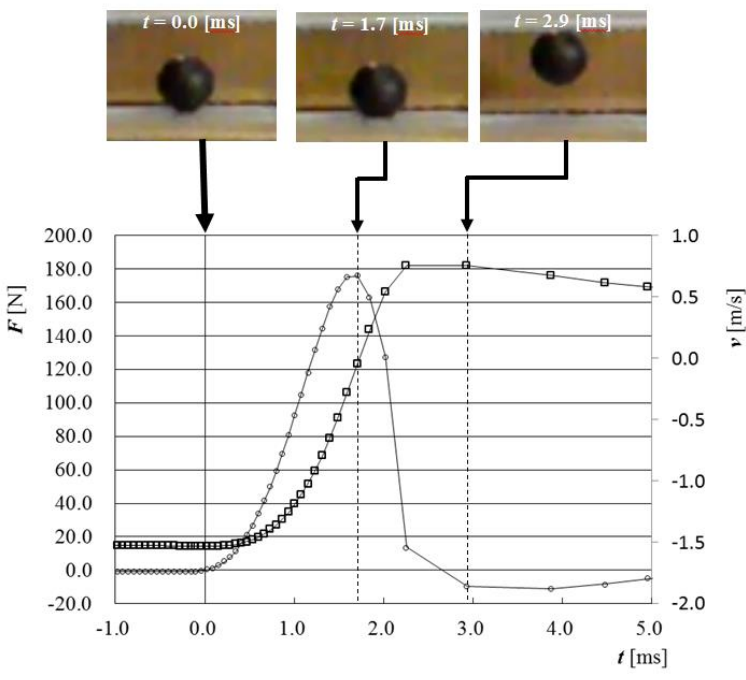

Fig. 5. Changes in impact force and velocity against time correlated with images taken by the high-speed camera.

Figure 5 shows the changes in impact force and velocity against time correlated with images taken by the high-speed camera.

\section{Discussion}

The sources of uncertainty in determining the instantaneous value of $F_{\text {gelsheet }}$ against time for all the 10 drop measurements are considered as follows:

Optical alignment: The major source of uncertainty in the optical alignment was the inclination of the $1 \mathrm{mrad}$ signal beam; this resulted in a relative uncertainty in the inertial force of approximately $5 \times 10^{-7}$, which is negligible

Mass calibration: The uncertainty in the mass measurement when using the electric balance was approximately $0.01 \mathrm{~g}$, which corresponds to $0.01 \%$ of the total mass of the impact force. This corresponds to $0.1 \mathrm{mN}$ when the applied force impact its maximum value $F_{\text {mas,max }}=$ $1.0 \mathrm{~N}$, which is negligible.

Acceleration due to gravity: The acceleration due to gravity $g$ is estimated to be $9.799 \mathrm{~m} / \mathrm{s}$ with an uncertainty of $0.01 \%$ which is negligible.

\section{Conclusions}

A method for testing the impact absorption measurement of a gel sheet by releasing the metal ball from drop height with the velocity of the center of gravity of the metal ball is proposed. The measurement is accurately measured using an optical interferometer with a sampling interval of approximately $1 \mathrm{~ms}$. The acceleration, displacement, and inertial force of the metal ball are calculated from the velocity. The force of impact and velocity against time was recorded using a high-speed camera for the interference stripe in real time.

\section{Acknowledgment}

This study was conducted in a fruitful achievement for collaboration between Gunma University and Kasem Bundit University. The author would like to thank Prof.Yusaku Fujii for useful discussions, Prof. Akihiro Takita for the valuable comments, and their graduate students for the experimental setup. Special thanks to Prof.Takao Yamaguchi gave us valuable comments on the material testing, especially, visco-elasticity material testing, and Prof.Tsuneaki Ishima provided the high-speed camera, we used.

\section{References}

(1) BJ. Briscoe, F. Motamedi, "The ballistic impact characteristics of aramid fabrics: the influence of interface friction", Wear, 158(1-2), 229-47, 1992.

(2) S. Bazhenov, "Dissipation of energy by bulletproof aramid frabrics”, J Mater Sci, 32(15), 4167-73, 1997.

(3) Bing Wang, Jian Xiong, Xiaojun Wang, Li Ma, Gui-Qi Zhang, Lin-Zhi Wu, and Ji-Cai Feng, "Energy absorption efficiency of carbon fiber reinforced polymer laminates under high velocity impact", Elsevier, 50, 140-148, 2013.

(4) S.S. Morye, P.J. Hine, R.A. Duckett, D.J. Carr, and I.M. 
Ward, "Modelling of the energy absorption by polymer composites upon ballistic impact.", Elsevier, 60, 2631-2642, 2000.

(5) Y. FUJII and T. YAMAGUCHI, "Proposal for material viscoelasticity evaluation method under impact load", Spring Science + Business Media, Inc., No.40, pp.4785-4790, 2004.

(6) Y. Fujii, "Proposal for a Step Response Evaluation Method for Force Transducers", Measurement Science \& Technology, 14, 1741-1746, 2003.

(7) Y. Fujii, "A Method for Calibrating Force Transducers Against Oscillation Force", Measurement Science \& Technology, 14, 1259-1264, 2003.

(8) Y. Fujii, "Microforce materials Tester", Review of Scientific Instruments, 76, 065111, 1-7, 2005.

(9) Y. Fujii, H. Fujimoto, R. Watanabe, and Y. Miki, "Balance for measuring mass under microgravity conditions", AIAA Journal, 39(3), 455-457, 2001

(10) Y. Fujii, "Towards Establishing Dynamic Calibration Method for Force Transducers", IEEE Transactions on Instrumentation and Measurement, 2009.

(11) Y. Fujii, "Microforce materials Tester", Review of Scientific Instruments, 76, 065111, 1-7, 2005.

(12) Yusaku Fujii, "Dynamic three-point bending tester using inertial mass and optical interferometer", Optics and Lasers in Engineering, 38, 305-318, 2002.

(13) Y. Fujii, T. Yamaguchi, and J.D.R. Valera, "Impact response measurement of a human arm", Society for Experimental Mechanics, 64-68, 2006.

(14) Y. Fujii, "Measurement of Impulse Response of Force Transducers", Review of Scientific Instruments, 72, 3108-3111, 2001.

(15) T. Bruns, R. Kumme, M. Kobusch, and M. Peters, "From Oscillation to Impact: The Design of a New Force Calibration Device at PTB”, Measurement, 32, 85-92, 2002.

(16) Y. Fujii, "Measurement of Force Acting on a Moving Part of a Pneumatic Linear Bearing", Review of Scientific Instruments, 74, 3137-3141, 2003.

(17) Li-Xin Xia, Run-Qin Liu, Sheng-Qing Yu, Bin Xie, Xiang-Ling Zhou, and Ling Guo, "Higher precision to estimate a phase via an optical interferometer with multiple passes.”, Elsevier B.V., 335, 129-132, 2015.

(18) Y. Fujii, "Dynamic Three-point Bending Tester Using Inertial Mass and Optical Interferometer", Optics and Lasers in Engineering, 38, 305-318, 2002.

(19) S. Bernhardt, M. Ramulu and A.S. Kobayashi, "Low-velocity Impact Response Characterization of a
Hybrid Titanium Composite Laminate", Transactions of the ASME, 129, pp.220-226, 2007.

(20) Ahmet S. Yigit and Andreas P. Christoforou, "Limits of asymptotic solutions in low-velocity impact of composite plates.”, Elsevier, 81, 568-574, 2007.

(21) Y. Park, R. Kumme, and D. Kang, "Dynamic Investigation of a Binocular Six-component Force-moment Sensor", Measurement Science and Technology, 13, 1311-1318, 2002.

(22) Naoyuki Koyama, "A method of step height measurement within the unambiguous range of two laser wavelengths interferometer.", Elsevier, 126, 313-316, 2015.

(23) Santosh Kumar, Ashish Bisht, Gurdeep Singh, Kuldeep Choudhary, and Divya Sharma, "Implementation of wavelength selector based on electro-optic effect in Mach-Zehnder interferometers for high speed communications.”, Elsevier B.V., 350, 108-118, 2015.

(24) Jiangtao Zhou, Yiping Wang, Changrui Liao, Bing Sun, Jun He, Guolu Yin, Shen Liu, Zhengyong Li, Guanjun Wang, Xiaoyong Zhong, and Jing Zhao, "Intensity modulated refractive index sensor based on optical fiber Michelson interferometer.", Elsevier, B208, 315-319, 2015.

(25) T. Takeda, K. Ishigami, K. Shintato, K. Nakajima, and A. Shimada, "The influence of impact object characteristics on impact force and force absorption by mouth guard material", Blackwell Munksgaard, 20, 12-20, 2004.

(26) T. Takeda, K. Ishigami, H.Jun, K. Nakajima, A. Shimada, and T. Ogawa, "The influence of the sensor type on the measured impact absorption of mouthguard material”, Blackwell Munksgaard, 20, 29-35, 2004.

(27) R. Araki, A. Takita, T. Ishima, H. Kawashima, N. Pornsuwancharoen, S. Punthawanunt, E. Carcasona and Y. Fujii, "Impact force measurement of a spherical body dropping onto a water surface", AIP Publishing LLC., 85, 075108, 2014.

(28) M.O.W. Rechardson and M.J. Wisheart, "Review of Low-Velocity Impact Properties of Composite Materials”, Composites, 27A, pp.1123-1131, 1996.

(29) D. Karagiozova and Norman Jones, "Dynamic effects on buckling and energy absorption of cylindrical shells under axial impact”, Elsevier, 39, pp.583-610, 2001.

(30) Y. Duan, M. Keefe, T.A. Bogetti, B.A. Cheeseman, and B. Powers, "A numerical investigation of the influence of friction on energy absorption by a high-strength fabric subjected to ballistic impact.", Elsevier, 32, 
1299-1312, 2006.

(31) Seong Sik Choen, Tae Seong Lim, and Dai Gil Lee, "Impact energy absorption characteristics of glass fiber hybrid composites.”, Elsevier, 46, 267-278, 1999.

(32) G.M. Nagel and D.P. Thambiratnam, "A numerical study on the impact response and energy absorption of tapered thin-walled tubes.", Elsevier, 46, 201-216, 2004.

(33) JD. Reid, "Towards the understanding of material property influence on automotive crash structures", Thin-Walled Struct, 24, pp.285-313, 1996.

(34) Y. Fujii, "A Method for Determining the Impact Force in Crash Testing", Academic Press, 14(6), 959-965, 1999.

(35) Huw Davies, Alexandre Assoune and Bill Courtney, "Pedestrian protection using a shock absorbing liquid (SALi) based bumper system", In $21^{\text {st }}$ International Technical Conference on the Enhanced safety of vehicles (ESV 2009), No. 09-0027, 2009.

(36) Haibo Yang, Fanzhu Li, Tung W. Chan, Li Liu and Liqun Zhang, "A simulation study on the effect of spring-shaped fillers on the viscoelasticity of rubber nanocomposite", Elsevier Ltd., 1359-8368, 2015.

(37) Y. Fujii, and T. Yamaguchi, "Method for Evaluating Material Viscoelasticity", Review of Scientific Instruments, 75, 119-123, 2004.

(38) Y. Fujii, and T. Yamaguchi, "Method for Evaluating Material Viscoelasticity", Journal of Materials Science, 40, 4785-4790, 2005.

(39) Y. Fujii, and K. Maru, "Self-correction method for dynamic measurement error of force sensors", Society for Experimental Mechanics, 35, 15-20, 2011.

(40) Y. Fujii, and J.P. Hessling, "Frequency estimation method from digitized waveform", Society for Experimental Mechanics, 33, 64-69, 2009. 